\title{
Randomized controlled trial comparing hyaluronic acid, platelet-rich plasma and the combination of both in the treatment of mild and moderate osteoarthritis of the knee- Letter to the Editor \& Author Response
}

\section{Letter to the Editor}

Patel $\mathrm{S}^{1}$, Dhillon $\mathrm{MS}^{1}$, Bansal $\mathrm{T}^{1}$

Dear Editor,

We read with great interest the article published online in your journal on $3^{\text {rd }}$ August 2016 by Lana et al entitled "Randomized controlled trial comparing hyaluronic acid, platelet-rich plasma and the combination of both in the treatment of mild and moderate osteoarthritis of the knee". ${ }^{[1]}$. First of all we would like to congratulate the authors on a beautifully designed and well executed study. PRP and hyaluronic acid are at present the forerunners in the race for treatment of osteoarthritis. While many studies have highlighted their effects individually, this is one of the earliest to highlight their synergistic effect in OA knee in humans. Orthobiologics may now be the evolving modality in the treatment of osteoarthritis, and we believe this will certainly be the future of treatment of osteoarthritis. Nevertheless, we would like to clarify certain points, and present our point of view pertaining to the article.

1) The authors have used high molecular weight hyaluronic acid (2.4-3.6 MDa) in concentration of $10 \mathrm{mg} / \mathrm{ml}$, ie $1 \% \mathrm{w} / \mathrm{v}$ (Eufflexa-Ferring 10mg/ml HA) and HA has been used with PRP in the ratio of 1:2.5. We would like to question and get calrifications about the rationale behind using this combination. Were any studies done for arriving at this specific combination? It is pertinent to note that it has been highlighted by some in vitro studies that PRP exerts a pure dilution effect on HA. Therefore, dilution in a ratio greater than $1: 1$, and the use of concentration below $1 \%$ affects the rheological properties of HA and may not be suitable. It has also been shown that low molecular weight HA enhances the synergistic effects when used in combination with PRP, and that Low molecular weight HA is better than using High molecular weight $\mathrm{HA}^{[2]}$.

2) Secondly, we would like to project our belief that it would have been more logical to give PRP injections first, followed by HA injections in an interval of one month ${ }^{[3]}$. This would prevent PRP and HA from potentially interfering with each other, but would still provide a synergistic effect.

3) Another point of note is that the authors have not mentioned as to how they arrived at the sample size required for their randomized trial. They are requested to provide clarification as to to which primary parameter (VAS versus WOMAC) was used in sample size calculation. The sample size of the study seems inadequate for it to be labelled as an RCT as per CONSORT guidelines. Considering the absence of a true control group (Saline Placebo), and as both HA and PRP are established as treatment modalities in relieving pain in Knee OA, the sample size should have been larger to demonstrate a significant difference between the treatment groups.

4) We would also like the authors to shed some light on the randomization protocol that was followed. Despite randomization, the groups were skewed with the HA group demonstrating significantly more WOMAC pain score in the baseline analysis of the groups. This non homogenosity of data leads to serious questions regarding randomization method. On the other hand, this could be a result of the small sample size.

5) We would also like to point out that blinding of patients is an important issue; clarification is needed as to how blinding was achieved in subjects evaluated, as PRP preparation involves drawing of blood. Hence, blinding in the HA group would have been difficult, and needs to be clarified.

Based on the positive results of the present study and other in vitro studies, we are hopeful that combinations of HA and PRP will turn out to be an effective therapy for OA knee in the future. However, many more well designed randomized control trails, with larger sample sizes, are needed to determine the ideal dosing schedules and combinations.

\footnotetext{
Author Names in full: Sandeep Patel ${ }^{1}$, M S Dhillon ${ }^{1}$, Tungish Bansal ${ }^{1}$
} 


\section{References}

1. Lana JF, Weglein A, Sampson S, Vicente EF, Huber SC, Souza CV, Ambach MA, Vincent H, Urban-Paffaro A, Onodera CM, Annichino-Bizzacchi JM. Randomized controlled trial comparing hyaluronic acid, platelet-rich plasma and the combination of both in the treatment of mild and moderate osteoarthritis of the knee. J Stem Cells Regen Med. 2016.

2. Russo F, D'Este M, Vadalà G, Cattani C, Papalia R, Alini M, Denaro V. Platelet rich plasma and hyaluronic acid blend for the treatment of osteoarthritis: Rheological and biological evaluation. PLoS One. 2016;11(6):e0157048.

3. Patel S, Dhillon MS, Bansal T. PRP and HA for Hip Osteoarthritis: Letter to the Editor. The American Journal of Sports Medicine. 2016;44(9):NP44.

\section{Corresponding Author}

Sandeep Patel, Department of Orthopaedics, Post Graduate Institute of Medical Education and Research, Chandigarh, India; Emailsandeepdrpatelortho@gmail.com

\section{Author response to Patel et al's Letter to the editor}

\section{Dear Editor,}

With reference to the Letter to the Editor regarding our manuscript, entitled "Randomized controlled trial comparing hyaluronic acid, platelet-rich plasma and the combination of both in the treatment of mild and moderate osteoarthritis of the knee", we have worked thoroughly to answer all questions raised. We are presenting below all of the answers questioned. We have prepared a list below that responds to the comments.

1. The authors have used high molecular weight hyaluronic acid (2.4-3.6 MDa) in concentration of $10 \mathrm{mg} / \mathrm{ml}$, ie $1 \% \mathrm{w} / \mathrm{v}$ (EufflexaFerring $10 \mathrm{mg} / \mathrm{ml} \mathrm{HA}$ ) and HA has been used with PRP in the ratio of $1: 2.5$. We would like to question and get calrifications about the rationale behind using this combination. Were any studies done for arriving at this specific combination? It is pertinent to note that it has been highlighted by some in vitro studies that PRP exerts a pure dilution effect on HA. Therefore, dilution in a ratio greater than $1: 1$, and the use of concentration below $1 \%$ affects the rheological properties of HA and may not be suitable. It has also been shown that low molecular weight HA enhances the synergistic effects when used in combination with PRP, and that Low molecular weight HA is better than using High molecular weight $\mathrm{HA}^{[2]}$.

Answer: We acknowledge this suggestion. The proportion between HA and PRP wasn't made thinking in rheological properties of HA. This association was made to decrease the levels of pro-inflammatory cytokines, which are present in PRP based on in vitro studies that compared use of synovial cells and chondrocytes in which the effects of PRP or HA on inflammation were compared by measuring TNF- $\alpha, I L-6$ and IL-8. A decrease in IL-6 was found only in HA cultures, and TNF- a was found in both. On the other hand, metalloproteinases were reduced when these cells were treated only with PRP. These results suggest that both treatments reduce inflammation by different mechanisms. The association of both products may infer advantages when injected ${ }^{[1]}$. Also, when the motility of synovial fibroblasts and tendon cells was evalated, it was verified that the cells that used the association between HA and PRP had better improvement in migration
(335\%) when compared to treatments alone [2].Also, the synergistic anabolic action of $H A$ and $P R P$ has been demonstrated in a $3 D$ arthritic neo-cartilage and ACLT-OA model. Indeed, the combination of $H A+P R P$ can synergistically promote cartilage regeneration and inhibit $O A$ inflammation ${ }^{[3]}$. Currently, there are reports which claim excellent results of the $H A+P R P$ association in Morton neuroma surgery and in the healing of pressure ulcers and surgical wounds ${ }^{[4]}$. However, these findings need confirmation by controlled trials, because only one study has assessed the superiority of the composite PRP/HA in the treatment of pressure ulcers, in comparison with HA or PRP, used alone. In osteoarthritis - Progress in Basic Research and Treatment, the ability to understand and control the factors that play a role in the therapeutic effect of $H A+P R P$ shall guide the optimization and design of the combination i.e. optimal ratio, molecular weight of HA, optimal PRP formulation. Regarding molecular weight, although it has been shown that low molecular weight HA (LMW HA) enhances the synergistic effects when used in combination with PRP, these conclusions are not supported by well-elucidated mechanisms. On the other hand, there are other studies in favor of High molecular weight HA (HMW HA,) which relies on the mechanical and biological effects of $H A$ alone. Therefore, the better molecular weight of HA for use with PRP in treatment of knee osteoarthritis is still controversial. Thus, our choice was based on relevant findings from the literature as follows:

Sato et al. (2014) investigated the effects of HA molecular weight on chondrogenic differentiation. They found that HMW HA (6 MDa) significantly promoted chondrogenic differentiation of ATDC5 cells in vitro, and suggested that HMWHA plays a significant chondroregulatory role in vivo ${ }^{[5]}$. HA also has been shown to have differential signaling based on its molecular weight. Macrophages exhibit phenotypic diversity permitting wide-ranging roles in maintaining physiologic homeostasis. In this context, Rayahin et al. (2015) studied the role of HA molecular weight on macrophage activation and reprogramming. Their observations suggest that macrophages undergo phenotypic changes dependent on molecular weight of HA that correspond to either (1) pro-inflammatory response for LMWHA or (2) pro-resolving response for HMW HA ${ }^{[6]}$. Smith and Gosh (1987) shown that in vitro synthesis of HA by human synovial fibroblasts is influenced by the concentration and molecular weight of HA in extracellular environment. Synovial fibroblasts derived from an osteoarthritic joint demonstrated the most marked response on exposure to exogenous $H A$, showing a stimulation of $H A$ synthesis with preparations of intermediate or $H M W>5 \times 10^{5} \mathrm{Da}$, in a concentration dependent manner. HA preparations with $M W<5 \times 10^{5}$ showed little or no effect except at high concentrations where a suppression of biosynthesis was observed ${ }^{[7]}$.

In a review article on the efficacy and safety of $H A$ in the management of osteoarthritis, Maheu et al. (2016) conclude that the current evidence available does not support a superiority of one kind of MW HA preparation over another, perhaps with the exception of a slightly lower efficacy for $L M W$ preparations versus intermediate and high MW HA shown in a single trial which requires further investigation ${ }^{[8]}$.

Recently, Saturveithan et al. (2016) reported a comparative study of the functional outcomes of intra-articular $H A+P R P$ vs. HA injections alone in patients with grade III and IV knee osteoarthritis. In this study, the authors justified the choice of HMW HA instead of LMW HA, based on the following reasons: HMW HA $\left(1.5 \times 10^{6} \mathrm{Da}\right)$ closely resembles the molecular weight of endogenous $H A\left(\sim 2 \times 10^{6}\right.$ $D a)$ in the extracellular matrix; studies have demonstrated that $H M W H A$ down regulates the gene expression of osteoarthritis associated cytokines and enzymes in fibroblast like synoviocytes; in addition, HMW HA has anti-inflammatory property and regulates the suppressor T cells for cell proliferation; supporting evidence also shows that the HMW HA typically resides longer in the synovial joint 
as compared to LMW HA. This property improves the efficacy of $H M W H A$ in inhibiting glycosaminoglycan release from the articular cartilage and results in better outcome in the long run. From the obtained results, the authors strongly suggested combination of intra-articular HA and PRP injection as an optional treatment modality in the treatment of grade III and IV knee osteoarthritis in terms of functional outcome and pain control for up to 6 months of duration when surgical treatment is not an option ${ }^{[9]}$.

In general, the literature shows that the number of injection needed is lower when HMW-HA is used, and this is not negligible advantage for the patients.

Regarding some studies that show the addition of PRP to HA exerts a pure dilution effect, our in vitro studies have shown that PRP activated with thrombin and calcium improves the rheological properties of HMW HA due to interactions with the fibrin network (data not published). The association 1:1 HA/PRP produces a typical gel behavior, with viscoelastic shear modulus twice the viscosity modulus throughout a wide range of frequencies. This is different from the behavior of a HA solution (1\%) that presents a cross-over point at low frequency. Thus, although an optimal concentration of the combination was not established, higher proportions of PRP in relation to HMW HA, should still keep adequate rheological properties.

2. Secondly, we would like to project our belief that it would have been more logical to give PRP injections first, followed by HA injections in an interval of one month. [3]. This would prevent PRP and HA from potentially interfering with each other, but would still provide a synergistic effect.

Answer: We acknowledge this suggestion. However, our idea was to verify the association of PRP + HA together and for this the design of the study had 3 groups (PRP, HA, and the combination PRP+HA). We think about using HA to inhibit the inflammatory process, through IL-6, IL-1, TNF- $\alpha$, which are present in PRP samples, and without thinking about the rheological property at this time. Currently, in the literature, there are some studies with this design, as that of Guo et al., (2016). In this study, the patients received a weekly intra-articular injection (lateral approach) of the compound PRP plus HA or only PRP for 3 weeks. The authors concluded that the association of PRP and HA is effective and safe in the management of patients suffering from mild to moderate OA. Although, no differences in functional outcomes were shown between the groups, there is a trend that PRP plus HA could obtain relatively better functional scores ${ }^{[10]}$. Dallari et al., (2016) published a paper for hip osteoarthritis in order to evaluate the treatment of HA, PRP and the association of both. The number of participants and treatment schedule were similar to our study. All patients received 3 consecutive intra-articular ultrasound-guided injections during outpatient surgery, 1 week apart, of $5 \mathrm{~mL}$ of autologous PRP (PRP group), $2 \mathrm{~mL}$ of HA (Hyalubrix $30 \mathrm{mg} / 2 \mathrm{~mL}$; Fidia Farmaceutici SpA) (HA group), or $7 \mathrm{~mL}$ of PRP+HA (PRP:HA = 5:2 mL) [11]. Although, there is a lack of few available studies, there is a scientific support in the study design employed in this work.

3. Another point of note is that the authors have not mentioned as to how they arrived at the sample size required for their randomized trial. They are requested to provide clarification as to to which primary parameter (VAS versus WOMAC) was used in sample size calculation. The sample size of the study seems inadequate for it to be labelled as an RCT as per CONSORT guidelines. Considering the absence of a true control group (Saline Placebo), and as both HA and PRP are established as treatment modalities in relieving pain in Knee OA, the sample size should have been larger to demonstrate a significant difference between the treatment groups.

Answer: We acknowledge this comment. The number of patients in our study was chosen based in the literature regarding RCT and the average value of 100 patients was obtained.

The primary analysis in this study (WOMAC and VAS) were not used for sample size calculation, but was used to see if there was significant difference between baseline and 360 days after application.

We would like to have a larger number of patients in this study. However, it is not easy to include patients and also due to high costs involved, especially with HA. The idea for the use of HA in one group was to compare as a standard therapy (like a control group), because in Brazil, it is not ethical in clinical trials to use a control group without any treatment (saline). In this way, the control group should be made with some type of treatment; the most used is conventional therapy. In the literature, we can see RCT with the same or less number of patients ${ }^{[1-14]}$, although the conclusions of these studies need to be tested in larger studies.

4. We would also like the authors to shed some light on the randomization protocol that was followed. Despite randomization, the groups were skewed with the HA group demonstrating significantly more WOMAC pain score in the baseline analysis of the groups. This non homogenosity of data leads to serious questions regarding randomization method; on the other hand this could be a result of the small sample size.

Answer: We acknowledge this comment. The randomization was made with a draw for papers in a glass jar. They were drawn according to group $A, B$ or $C$. There were 120 papers, 40 in each group. We observed the difference in the group of HA. However all the variables that imply the randomization of patients such as: race, grade of Kellgrew-Lawrence, body mass index, the WOMAC were used after the randomization, and not used for the randomization of our patients. The maximum and minimum values of the groups of WOMAC pain were similar among the groups.

5. We would also like to point out that blinding of patients is an important issue; clarification is needed as to how blinding was achieved in subjects evaluated, as PRP preparation involves drawing of blood. Hence blinding in the HA group would have been difficult, and needs to be clarified.

Answer: We acknowledge this comment. All of the patients from this study had blood collected, independently of the group of treatment. If the patients were from the HA group, the blood was discarded. The evaluation of the patients (WOMAC, VAS) was made with a blind appraiser, without any intervention in the analysis.

Sincerely,

Jose Fabio Santos Duarte Lana et al., Bone and Cartilage Institute, Instituto do Osso e da Cartilagem (IOC), Avenida Presidente Kennedy, número 1386, Salas 26, 28 e 29, Indaiatuba, São Paulo, Brazil, Zip Code: 13334-170; Email: josefabiolana@gmail.com 


\section{References}

1. Sundman EA, Cole BJ, Karas V, Valle CD, Tetreault MW, Mohammed HO, Fortier LA. The anti-inflammatory and matrix restorative mechanisms of platelet-rich plasma in osteoarthritis. Am. J. Sports Med. 2014; 42(1): 35-41.

2. Anitua E, Sanchez M, De la Fuente M, Zalduendo MM, Orive G. Plasma rich in growth factors (PRGF-Endoret) stimulates tendon and synovial fibroblasts migration and improves the biological properties of hyaluronic acid. Knee Surg.Traumatol. Arthrosc. 2012; 20 (9): 1657-65.

3. Chen WH, LO WC, Hsu WC, Wei HJ, Liu HY, Lee CH, Tina Chen SY, Shieh YH, Williams DF, Deng WP. Synergistic anabolic actions of hyaluronic acid and plateletrich plasma on cartilage regeneration in osteoarthritis therapy. Biomaterials 2014; 35(36): 9599-607.

4. De Angelis, Lucarini L, Orlandi F, Agovino A, Migner A, Cervelli V, Izzo V, Curcio C. Regeneratibe surgery of the complication with Morton's neuroma surgery: Use of platelet rich plasma and hyaluronic acid. Int. Wound $\mathrm{J}$. 2013; 10(4): 372-6.

5. Sato E, Ando T, Ichikawa J, Okita G, Sato N, Wako M, Ohba T, Ochiai S, Hagino T, Jacobson R, Haro H. High molecular weight hyaluronic acid increases the differentiation potential of murine chondrocytic ATDC5 cells line. J. Orthop. Res. 2014; 32(12): 1619-27.

6. Rayahin JE, Buhrman JS, Zhang Y, Koh TJ, Gemeinhart RA. High molecular weight hyaluronic acid differentially influence macrophage activation. ACS Biomater. Sci. Eng. 2015; 1(7): 481-93.

7. Smith MM, Ghosh P. The synthesis of hyaluronic acid by human synovial fibroblasts is influenced by the nature of the hyaluronate in the extracellular environment. Rheumatol. Int. 1987; 7(3): 113-22.

8. Maheu E, Rannou F, Reginster JY. Efficacy and safety of hyaluronic acid in the management of osteoarthritis: Evidence from real-life setting trials and surveys. Semin. Arthritis Rheum. 2016; 45(4 Suppl): s28-33.
9. Saturveithan C, Premganesh G, Fakhrizzaki S, Mahathir M, Karuna K, William H, Akmal H, Sivapathasundaram N, Jaspreet K. Intra-articular hyaluronic acid (HA) and platelet rich plasma (PRP) injection versus Hyaluronic acid (HA) injection alone in patients with grade III and IV knee osteoarthritis (OA): A restrospective study on functional outcome. Malays Orthop. J. 2016; 10(2): 35-40.

10. Guo Y, Yu H, Yuan L, Yao S, Yu H, Wang P, Lv H, Li W, Sun. Treatment of knee osteoarthritis with platelet-rich plasma plus hyaluronic acid in comparison with plateletrich plasma only. Int. J. Clin. Exp. Med. 2016; 9(6): 1208512090.

11. Dallari D, Stagni C, Rani N, Sabbioni G, Pelotti P, Torricelli P, Tschon M, Giavaresi G. Ultrasound-guided injection of platelet-rich plasma and hyaluronic acid, separately and in combination, for hip osteoarthritis: a randomized controlled study. Am. J. Sports Med. 2016; 44(3): 664-71.

12. Vaquerizo V, Plasencia MA, Arribas I, Seijas R, Padilla S, Orive G, Anitua E. Comparison of intra-articular injections of plasma rich in growth factors (PRGF-Endoret) versus durolane hyaluronic acid in the treatment of patients with symptomatic osteoarthritis: a randomized controlled trial arthroscopy. Arthroscopy. 2013; 29(10): 1635-1643.

13. Patel S, Dhillon MS, Aggarwal S, Marwaha N, Jain A. Treatment with platelet-rich plasma is more effective than placebo for knee osteoarthritis: a prospective, double-blind, randomized trial. Am. J. Sports Med. 2013; 41(2): 356-364.

14. Gosens T, Peerbooms JC, Laar WV, den Oudsten BL. Ongoing positive effect of platelet-rich plasma versus corticosteroid injection in lateral epicondylitis: a doubleblind randomized controlled trial with 2-year follow-up. Am. J. Sports Med. 2011; 39: 1200-1208. 